\title{
Refinement and Proof Based Development of Systems Characterized by Continuous Functions
}

\author{
Guillaume Babin ${ }^{1(\varpi)}$, Yamine Aït-Ameur ${ }^{1}$, Shin Nakajima ${ }^{2}$, and Marc Pantel $^{1}$ \\ 1 Université de Toulouse; IRIT / INPT-ENSEEIHT, \\ 2 Rue Charles Camichel, Toulouse, France \\ guillaume.babin@irit.fr, \{yamine,marc.pantel\}@enseeiht.fr \\ 2 National Institute of Informatics, 2-1-2 Hitotsubashi, Chiyoda-ku, Tokyo, Japan \\ nkjm@nii.ac.jp
}

\begin{abstract}
The specification of cyber-physical systems usually relies on continuous functions over dense real numbers whereas their implementation is discrete. Proving the correctness of the discrete implementation with respect to the continuous specification remains a challenge in the presence of dense real numbers. In this paper, we propose a refinementbased formal method, relying on Event-B, for such developments. We illustrate our proposal with the development of a simple stability controller for a generic plant model. The continuous function that models the system behavior is refined as a discrete model of the same kind preserving stability expressed as a safety invariants of the continuous model. The obtained discrete model uses discrete time (instants modeled on $\mathbb{N}$ ), whereas the continuous model is based on dense time (on $\mathbb{R}$ ). The Rodin Platform, together with the Theory plug-in handling the Real datatype and its properties supported the whole developments and proofs.
\end{abstract}

Keywords: Continuous and discrete behaviors - Dense real numbers · Correct-by-construction • Formal methods • Proved refinements • Event-B

\section{Introduction}

According to Lee [20], cyber-physical systems (CPS) are defined as integrations of computation, networking, and physical processes. Embedded computers and networks monitor and control the physical processes, with feedback loops where physical processes affect computations and vice versa. Most of the time, a software part (the controller) drives the physical part (the plant) through a loop involving sensors and actuators. The CPS plant behavior is given by dense time continuous functions solution of differential equations. The CPS controller behavior is specified by continuous functions over dense time. The CPS software implements a discretization of these functions in order to control the CPS plant. This discretization proof is a key challenge in the CPS correctness proof. 
In the past years, several approaches relying on formal methods, like Hybrid automata [17] and model checking [5], have been set up to describe the behavior of the software controllers. Our proposal focuses on the verification of correct controllers obtained after discretization.

This paper show how proof and refinement based approaches handle the development of a correct-by-construction discrete controller starting from a dense time continuous function specification of the continuous controller. A complete incremental development relying on a theory of reals is conducted to synthesize a correct discretization of a continuous function. The approach exploits an axiomatization of mathematical reals. It maintains a safety invariant characterizing the physical plant of the studied system. Such invariant defines a safety envelope (which we called safety corridor) modeling a stability property in which the system must evolve i.e. for a continuous function $f$, we write $\forall t \in \mathbb{R}^{+}, f(t) \in[m, M]$ where $t$ is a dense time parameter and the reals $m$ and $M$ define minimum and maximum values in $\mathbb{R}^{+}$ensuring a correct behavior of the physical plant. In general, these values come from the physics of the studied system. The Event-B method is used to handle such formal developments. We illustrate our proposal with the development of a simple stability controller for a generic plant model.

This paper is structured as follows. Section 2 overviews the addressed problem of discretization. Section 3 summarizes the Event-B method. Sections 4 and 5 are the core of our proposal: the refinement strategy for any continuous function together with the corresponding requirements are given in section 4 while the complete Event-B development handling these requirements is provided in section 5 . Related works and possible applications are sketched in section 6 . The conclusion and some perspectives are given in the end.

\section{Discretization of Continuous Functions}

The behavior of many systems can be characterized by three states: the initial boot, the nominal behavior, and the final halt. Several CPS involving physical plants and software controllers follow this pattern such as energy production systems, smart systems, medical systems, etc. These systems are usually modeled by differential equations specifying dense time continuous functions. In order to control their behavior, one has first to discretize these continuous functions. The main safety property concerns stability where the function values shall be maintained inside a safety envelope i.e. an interval of correct values (called corridor).

The correct implementation of such continuous functions is a key point in ensuring the CPS safety. These ones shall be discretized in a correct manner that guarantees that the discrete behavior simulates the continuous one. In other words, the continuous states existing between two observed consecutive states of the discretization are also in the safety corridor. To achieve this goal, we follow a correct-by-construction approach based on a formal development of any continuous function discretization, making our development reusable and scalable. The approach relies on refinement and on the preservation of invariants. Discretization information are incrementally added while moving from the 
continuous level to the discrete one. Event-B [1] and the Rodin Platform [2] have been set up to handle the developments.

\section{The Event-B Method}

An Event-B model [1] (see Table 1) is defined in a MACHINE. It encodes a state transition system which consists of: variables declared in the VARIABLES clause to represent the state; and events declared in the EVENTS clause to represent the transitions (defined by a Before-After predicate BA) from one state to another.

Table 1. Structure of Event-B machines

\begin{tabular}{|l|l|}
\hline CONTEXT & MACHINE \\
$c t x t_{\text {}} i d_{-2}$ & machine_id_2 \\
EXTENDS & REFINES \\
$c t x t_{-} i d_{-} 1$ & machine_id_1 \\
SETS & SEES \\
$s$ & $c t x d_{-} i d_{-} 2$ \\
CONSTANTS & VARIABLES \\
$c$ & $v$ \\
AXIOMS & INVARIANTS \\
$A(s, c)$ & $I(s, c v)$ \\
THEOREMS & THEOREMS \\
$T_{c}(s, c)$ & $T_{m}(s, c, v)$ \\
END & VARIANT \\
& $V(s, c, v)$ \\
& EVENTS \\
& Event $e v t \triangleq$ \\
& any $x$ \\
& where $G(s, c, v, x)$ \\
& then \\
& end $: \mid B A\left(s, c, v, x, v^{\prime}\right)$ \\
& END \\
\hline
\end{tabular}

Table 2. Generated proof obligations for an Event-B model

\begin{tabular}{|c|c|}
\hline Theorems & $\begin{array}{l}A(s, c) \Rightarrow T_{c}(s, c) \\
A(s, c) \wedge I(s, c, v) \\
\quad \Rightarrow T_{m}(s, c, v)\end{array}$ \\
\hline $\begin{array}{l}\text { Invariant } \\
\text { preservation }\end{array}$ & $\begin{array}{l}A(s, c) \wedge I(s, c, v) \\
\quad \wedge G(s, c, v, x) \\
\quad \wedge B A\left(s, c, v, x, v^{\prime}\right) \\
\quad \Rightarrow I\left(s, c, v^{\prime}\right)\end{array}$ \\
\hline $\begin{array}{l}\text { Event } \\
\text { feasibility }\end{array}$ & $\begin{array}{l}A(s, c) \wedge I(s, c, v) \\
\quad \wedge G(s, c, v, x) \\
\quad \Rightarrow \exists v^{\prime} . B A\left(s, c, v, x, v^{\prime}\right)\end{array}$ \\
\hline $\begin{array}{l}\text { Variant } \\
\text { progress }\end{array}$ & $\begin{aligned} A(s, c) & \wedge I(s, c, v) \\
& \wedge G(s, c, v, x) \\
& \wedge B A\left(s, c, v, x, v^{\prime}\right) \\
& \Rightarrow V\left(s, c, v^{\prime}\right)<V(s, c, v)\end{aligned}$ \\
\hline
\end{tabular}

A model also holds INVARIANTS and THEOREMS to represent its relevant properties. A decreasing VARIANT may introduce convergence properties when needed. An Event-B machine is related, through the SEES clause to a CONTEXT which contains the relevant sets, constants axioms, and theorems. The refinement capability [4], introduced by the REFINES clause, decomposes a model (thus a transition system) into another transition system containing more design decisions thus moving from an abstract level to a less abstract one. New variables and new events may be introduced at the refinement level. In a refinement, the invariant shall link the variables of the refined machine with the ones of the refining machine. A gluing invariant is introduced for this purpose. It preserves the proved properties and supports the definition of new ones.

Once an Event-B machine is defined, a set of proof obligations is generated. They are submitted to the prover embedded in the RODIN platform. Proof obligations associated to an Event-B model are listed in Table 2, here the prime notation is used to denote the value of a variable after an event is triggered. More details on proof obligations can be found in [1]. 
Use of Reals in Event-B. A recent evolution of the Event-B method allows to extend it with theories [13] similar to algebraic specifications. In the Rodin Platform, this evolution is provided by the Theory plugin for Rodin [3]. We need to model and reason on dense reals. We rely on the theory for reals and continuous functions, written by Abrial and Butler ${ }^{1}$. It provides a dense mathematical REAL datatype with arithmetic operators, axioms and proof rules.

Remark. From a tool point of view, the use of reals with the Theory plugin for Rodin introduces constants like zero and operators defined on the REAL datatype like smr for $<$, gtr for $>$ or leq for $\leq$. Casting operators need to be defined in order to work with other data types. These ones are used when discretizing continuous representations by refinement (see section 5.3).

\section{Refinement Strategy}

The mathematical model and the specification of the system behavior are sketched below. Following the approach defined in [23], the adopted refinement strategy consists in three steps: first, as shown in figure 1, we use three states to define a simple abstract controller that models the system; then, in a first refinement, we introduce a continuous controller characterizing its behaviors with a continuous function; finally, a second refinement builds a discrete controller.

\subsection{The Illustrating System}

The considered system goes through three phases. Figure 1 depicts its general behavior. First, it is booted (transition boot from state 1 to 2). After a while, once in state 2, it becomes operational in a nominal mode (run transition). Then, it stays a given amount of time in the nominal or running mode. When in nominal mode, it may be halted (stop transition from state 2 to state 3 ) for example in case a failure occurs or for maintenance purposes. This behavior is the one of a simple abstract system controller. When booting, the system cannot be stopped until it reaches the nominal mode. Other complex behavior scenarios can be defined with more complex transition systems.

In order to guarantee a correct behavior of the system, the previously defined controller shall fulfill the requirements from table 3 . These ones ensure that the system is correctly controlled. For example, an energy production system requires that the power produced by a given system belongs to a specific interval or a pacemaker must be pacing when a sensed signal belongs to another specific interval.

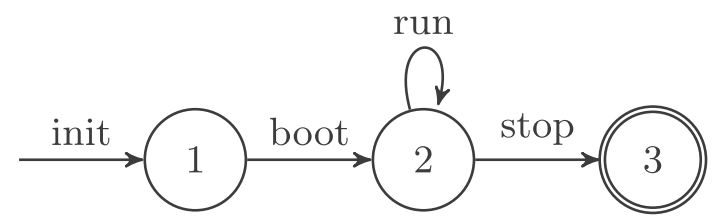

Fig. 1. Controller Automaton

\footnotetext{
${ }^{1}$ http://wiki.event-b.org/index.php/Theory_Plug-in\#Standard_Library
} 
Table 3. Requirements at the top level

\begin{tabular}{|l|c|}
\hline $\begin{array}{l}\text { At any time, the output value of the controlled system shall be less or } \\
\text { equal to } M \text { in any mode. }\end{array}$ & Req.1 \\
\hline $\begin{array}{l}\text { At any time, the output value of the controlled system shall belong to an } \\
\text { interval }[m, M] \text { in running mode. }\end{array}$ & Req.2 \\
\hline $\begin{array}{l}\text { At any time, if any future output value of the controlled system does not } \\
\text { belong to an interval }[m, M], \text { then the system is stopped. }\end{array}$ & Req. 3 \\
\hline
\end{tabular}

\subsection{Continuous Controller}

After modeling the system at an abstract level using three states, we introduce the continuous controller through the definition of a continuous function of the dense time $f: \mathbb{R}^{+} \rightarrow \mathbb{R}^{+}$corresponding to the behavior of the system.

The requirements identified in the previous section, are rewritten (refined) to handle the introduced continuous function behavior (see table 4).

Table 4. Requirements at the first refinement

\begin{tabular}{|l|c|}
\hline$m<M$ & Req.0 \\
\hline$\forall t \in \mathbb{R}^{+}, f(t) \leq M$ & Req.1 \\
\hline$\forall t \in \mathbb{R}^{+}$, state $(t)=2 \Rightarrow f(t) \in[m, M]$ & Req.2.1 \\
\hline$\forall t_{1}, t_{2} \in \mathbb{R}^{+}, t_{1}<t_{2}$, state $\left(t_{1}\right)=2 \wedge f\left(t_{2}\right) \in[m, M] \Longrightarrow$ state $\left(t_{2}\right)=2$ & Req.2.2 \\
\hline$\forall t_{1}, t_{2} \in \mathbb{R}^{+}, t_{1}<t_{2}$, state $\left(t_{1}\right)=2 \wedge f\left(t_{2}\right) \notin[m, M] \Longrightarrow$ state $\left(t_{2}\right)=3$ & Req. 3 \\
\hline
\end{tabular}

The control action over this system is a simple one. It consists in shutting down the system if the value of $f$ goes out of range. The obtained continuous controller corresponds to a refinement of the abstract one from the previous section, it is described by a hybrid automaton [17]. We are aware that the control actions of the defined system are very simple. Our objective is to show how a controller (characterized by a simple state transition system) and a physical plant (characterized by a continuous function) can be formally integrated into a single Event-B formal development encoding incrementally a hybrid automaton.

The previously described behavior is depicted by the graph in figure 2(a). The system is initialized (at point $A$ corresponding to the transition init to enter state 1 ). It reaches the running mode state at point $B$ (corresponding to the event boot and entering state 2 ). The system stays in the safety corridor (between $m$ and $M$ in state 2). When point $C$ is reached, the controller switches its state from state 2 to state 3 by the transition stop in order to prevent $f$ from going over the threshold $M$. The system is then halted to reach point $D$ (corresponding to state 3 ).

\subsection{Discrete Controller}

In order to implement the previous controller, we need to discretize the observation of the system behavior. In practice, when using computers to implement such controllers, time is observed according to specific clocks and frequencies. In other words, observations are discrete and depend on the available clocks. 
Therefore, it is mandatory to define a correct discretization of time that preserves the continuous behavior introduced previously. This preservation entails the introduction of other requirements on the defined continuous function. Note that, in practice, these requirements correspond to requirements issued from the physical plant.

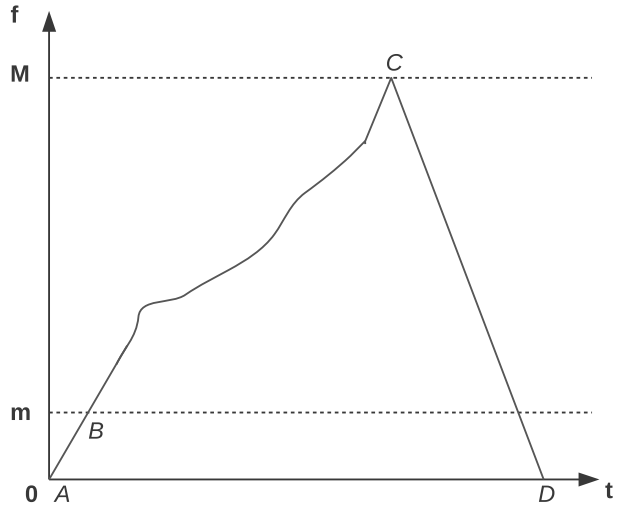

(a) Continuous controller

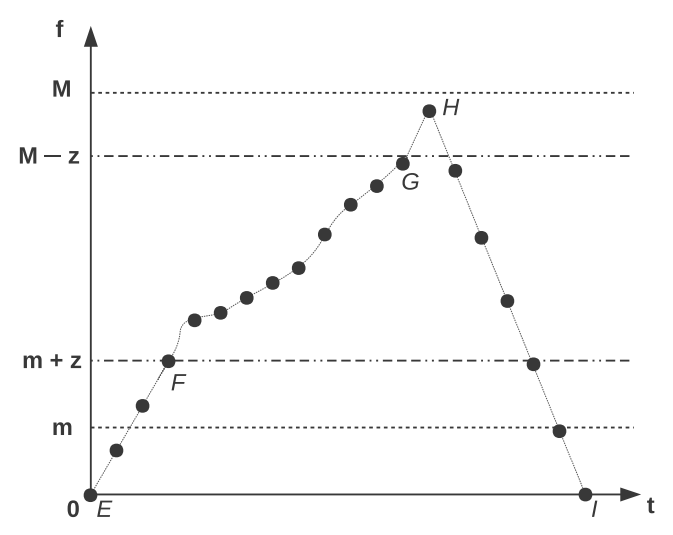

(b) Discrete controller

Fig. 2. Examples of the evolution of the function $f$

It is mandatory to introduce a margin allowing the controller to anticipate the next observable behavior before incorrect behavior occurs. Let $z$ be this margin. $z$ is defined such that the derivation of the function $f$ between two observed consecutive instants $t_{i}$ and $t_{i+1}$ shall not be greater than $z$. Formally, this is written as $z \geq \max _{i \in \mathbb{N}}\left|f\left(t_{i}\right)-f\left(t_{i+1}\right)\right|$. We assume that a value for $z$ exists (even if it is not the optimal one), it is obtained from the physical properties of the system. This means, that we need to identify the duration $\delta t$ defining the amount of time between two consecutive states observed by the discrete controller. As a consequence, we write $z \geq \max _{t \in \mathbb{R}^{+}}|f(t)-f(t+\delta t)|$. In order for the problem to be well-defined, $\delta t$ must be small enough so that the property $m+z<M-z$ holds. The set $\mathbb{D}$ of observation instants can be defined as $\mathbb{D}=\left\{t_{i} \mid t_{i} \in \mathbb{R} \wedge i \in \mathbb{N} \wedge t_{0}=0 \wedge t_{i+1}=t_{i}+\delta t\right\}$ and rewritten as $\mathbb{D}=\left\{t_{i} \mid t_{i} \in \mathbb{R} \wedge i \in \mathbb{N} \wedge t_{0}=0 \wedge t_{i}=i \times \delta t\right\}$.

As a consequence of this definition, the safety corridor becomes the interval $[m+z, M-z]$. Moreover, it becomes possible to observe, in the running mode,

Table 5. Requirements at the second refinement

\begin{tabular}{|c|c|}
\hline$z>0 \wedge m+z<M-z$ & Req.0 \\
\hline$\forall t_{i} \in \mathbb{D}, f\left(t_{i}\right) \leq M$ & Req.1 \\
\hline$\forall t_{i} \in \mathbb{D}$, state $\left(t_{i}\right)=2 \Rightarrow f\left(t_{i}\right) \in[m+z, M-z]$ & Req.2.1 \\
\hline $\begin{aligned} & \forall t_{i} \in \mathbb{D}, \text { state }\left(t_{i}\right)=2 \wedge f\left(t_{i}+\delta t\right) \in[m, M] \Longrightarrow \operatorname{state}\left(t_{i}+\delta t\right)=2 \\
\Leftrightarrow & \forall t_{i} \in \mathbb{D}, \text { state }\left(t_{i}\right)=2 \wedge f\left(t_{i+1}\right) \in[m, M] \Longrightarrow \operatorname{state}\left(t_{i+1}\right)=2 \\
\Leftrightarrow & \forall n \in \mathbb{N}, \operatorname{state}(n \delta t)=2 \wedge f((n+1) \delta t) \in[m, M] \Longrightarrow \operatorname{state}((n+1) \delta t)=2\end{aligned}$ & Req. 2.2 \\
\hline $\begin{array}{c}\forall t_{i} \in \mathbb{D}, \text { state }\left(t_{i}\right)=2 \wedge f\left(t_{i}+\delta t\right) \notin[m+z, M-z] \Longrightarrow \text { state }\left(t_{i}+\delta t\right)=3 \\
\Leftrightarrow \forall t_{i} \in \mathbb{D}, \operatorname{state}\left(t_{i}\right)=2 \wedge f\left(t_{i+1}\right) \notin[m+z, M-z] \Longrightarrow \text { state }\left(t_{i+1}\right)=3 \\
\Leftrightarrow \forall n \in \mathbb{N}, \operatorname{state}(n \delta t)=2 \wedge f((n+1) \delta t) \notin[m+z, M-z] \\
\Longrightarrow \operatorname{state}((n+1) \delta t)=3\end{array}$ & Req. 3 \\
\hline
\end{tabular}


two consecutive instants $t_{i}$ and $t_{i+1}$ such that $f\left(t_{i}\right) \in[m+z, M-z]$ and $f\left(t_{i+1}\right) \notin$ $[m+z, M-z] \wedge f\left(t_{i+1}\right) \in[m, M]$. This condition characterizes an exit from the safety corridor and thus the condition to stop the system and move to a stopping mode. Again, the previous requirements are refined to consider the discretization of time, using the two new parameters $z$ and $\delta t$, and $\mathbb{D}$ (Table 5).

The safety margin $z$ is defined such that if $f(n \delta t)$ is in $[m+z, M-z]$ then the value of $f$ observed by the controller, $f((n+1) \delta t)$, is in $[m, M]$. The definition of this discretization guarantees that Req.2.1 is fulfilled until the next value due to $\forall n \in \mathbb{N}, \quad \forall t \in[n \cdot \delta t,(n+1) \cdot \delta t], \quad|f(t)-f(n \delta t)| \leq z$. If the monitor observes a value in $[m, m+z[$ or in $] M-z, M]$, it shuts the system down because in the next step, the value might be out of range (Req. 3).

\subsection{Top-Down Refinement}

According to the previous definitions, the refinement starts from a generic definition of the system with the three identified events. The first refinement introduces the continuous function and the corresponding requirements of table 4 . We start with a continuous model $M_{c}$ of the system, describing the complete relevant physical behavior of the system. Then a second refinement defines the discrete model $M_{d}$ of the behavior correctly glued with the continuous one. Here, the refined requirements of table 5 are taken into account. Gluing invariants, formalizing the refined requirements, are introduced in order to preserve the proofs and the behavior of the abstraction. When proving the refinement, we demonstrate that our discrete model is a correct implementation of the desired continuous behavior (the specification).

To summarize, in $M_{c}$, the continuous function $f_{c}: \mathbb{R} \longrightarrow \mathbb{R}$ is considered. In $M_{d}$, we introduce a discrete function $f_{d}: \mathbb{N} \longrightarrow \mathbb{R}$, where $i \in \mathbb{N}$ is an instant and $\delta t$ is the time discretization interval duration. The functions $f_{d}$ and $f_{c}$ are glued by the following property: $\forall n \in 0 . . i, f_{c}(n \times \delta t)=f_{d}(n)$.

\subsection{About Modeling of Time}

In order to reduce the complexity of the proof of the discretization refinement corresponding to the introduction of $f_{d}$, we have split the behavior of $f_{c}$ during an $i^{\text {th }}$ discrete macro step $\left[t_{i},\left(t_{i}+\delta t\right)\right]$ into three kinds of smaller discrete micro steps (see figure 3). For example, at the running state (or nominal phase), we define the following micro steps.

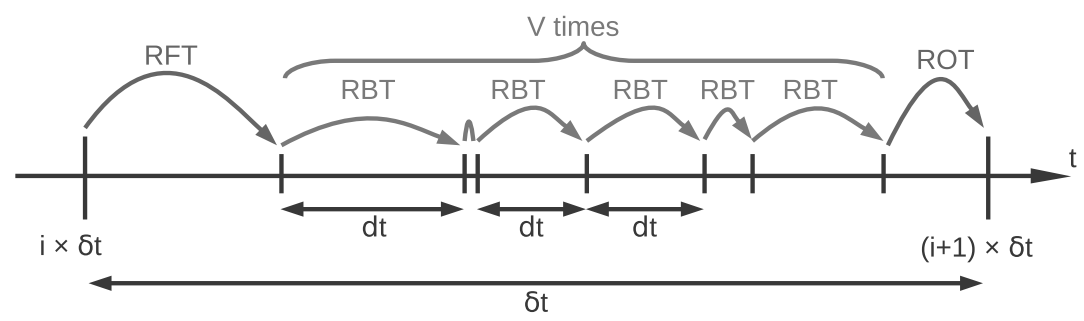

Fig. 3. Collapsing continuous time micro steps into a discrete time macro step 
1. RFT: run from tick is the first micro step inside a macro step starting at a tick (a discrete time $t_{i}=i \times \delta t$ ). Its duration is strictly smaller than $\delta t$.

2. RBT: run between ticks is a micro step strictly in the macro step (not the first nor the last micro step in a macro step). Its duration is denoted $d t>0$. A macro step contains $V$ occurrences of such micro steps.

3. ROT: run on ticks is the last micro step in the macro step.

The Zeno problem is avoided by guaranteeing that the number of micro steps of type RBT is finite, and that $d t>0$. From a modeling point of view, it will be formalized as a decreasing variant (natural number $V$ in $\mathbb{N}$ ). The trace of micro steps between $t_{i}$ and $t_{i+1}=t_{i}+\delta t$ is defined as RFT (RBT) $V$ ROT.

Our Event-B models introduce events aligned with these macro and micro steps either in the continuous case of in the discrete one.

\section{A Formal Development of a Discrete Controller with Event-B}

Our developments expressed within Event-B follow exactly the refinement strategy defined in section 4. According to [23], three development steps have been used. Contexts and machines are defined according to figure 4.

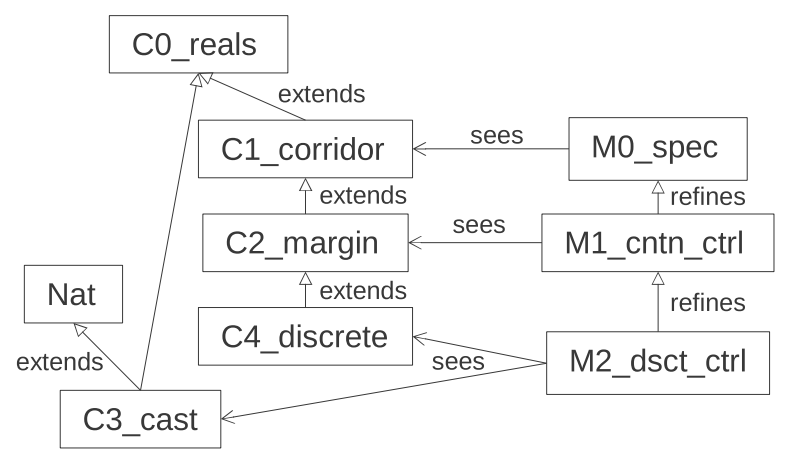

Fig. 4. Project structure

\subsection{Abstract Machine: The Top-Level Specification}

The top-level specification introduces the abstract controller with three events according to figure 1.

Needed Theories. To be able to handle real numbers and the corresponding theory, we have defined the context C0_reals which uses the theory defining reals. Listing 1.1 gives an extract of this context with axioms and theorems.

Several other axioms and theorems have been defined and proved. We show an extract of this theory. As mentioned in section 3 specific operators for manipulating reals are used. 


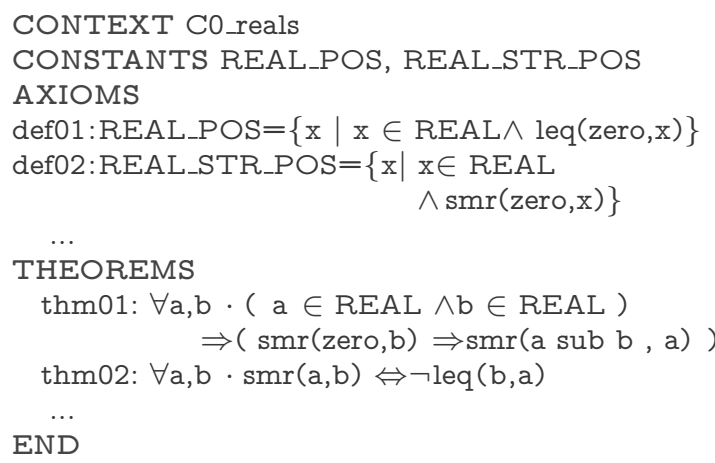

Listing 1.1. Part of context C0_reals
CONTEXT C1_corridor

EXTENDS C0_reals

CONSTANTS $\mathrm{m}, \mathrm{M}$

AXIOMS

axm01: $\mathrm{m} \in \mathrm{REAL}$ _STR_POS

axm02: $M \in R E A L \_S T R \_P O S$

$\operatorname{axm} 03: \operatorname{smr}(\mathrm{m}, \mathrm{M})$

END

Listing 1.2. Part of context C1_corridor

A second context defines the safety corridor with the values of $m$ and $M$. Listing 1.2 defines this context C1_corridor extending the context C0_reals.

The Top-Level Event-B Machine. It defines the global continuous values issued from the controlled system. The machine introduces the invariant inv03, guaranteeing Req.1 and Req.2.1 stating that in running mode (identified by active=true), the continuous value (defining the values of a continuous function introduced in the first refinement) $\mathrm{fv}$ shall be correct. This machine also models the abstract controller with three events boot, run and stop corresponding to the transition system of figure 1 . These events manipulate $f v$ the real positive value corresponding to the current continuous value.

Listing 1.3 gives an extract of the top specification machine M0_spec. To keep this paper in a reasonable length, only details for the event run are given ${ }^{2}$. Therefore, the Req. 3 will not explicitly be handled in this paper, it mainly concerns the stop event.

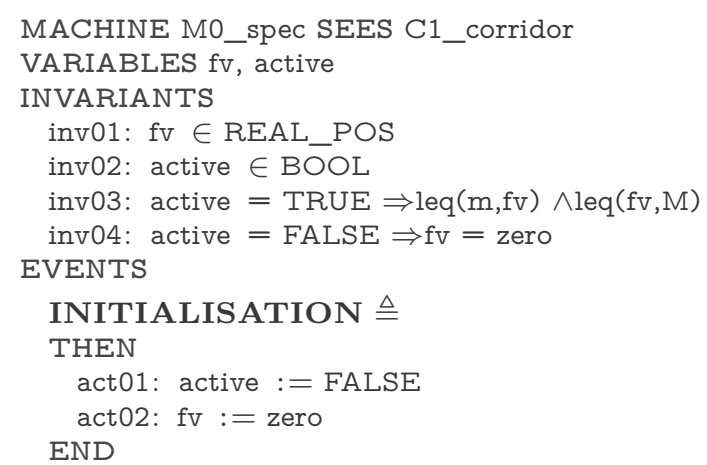

Listing 1.3. Extract of machine M0_spec

\footnotetext{
2 The complete Event-B developments can be downloaded from http://babin.perso.enseeiht.fr/r/SETTA2015EventBModels.pdf
} 


\subsection{The First Refinement: Introducing Continuous Functions}

Needed Theories. As shown on figure 4, the context C2_margin introducing the margin $\mathrm{z}$ is defined. Note that axm02 corresponds to the requirement Req.0.

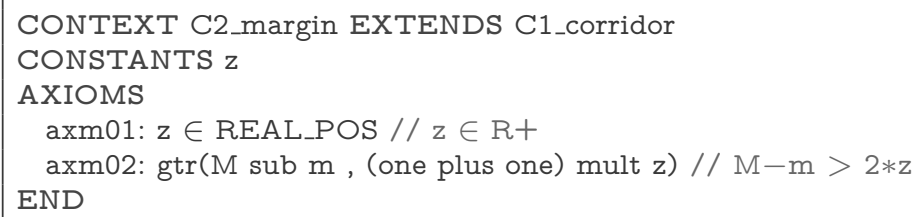

Listing 1.4. Extract of context C2_margin

The Event-B First Refinement with Continuous Functions. The first refinement M1_cntn_ctrl of the controller explicitly introduces

- the continuous function $f c$ producing the values $f v$ of the abstract machine and the corresponding invariant prop01

- dense time with the current instant noted now

- an important invariant glue01 gluing the continuous values of the abstraction with the continuous function defined on dense time $f \mathrm{v}=\mathrm{fc}$ (now)

- the variable active_t recoding the dense time where the system enters a running mode and the corresponding invariants glue02, glue03 and glue04 gluing the behavior of active_t with the active boolean variable.

The events of the M1_cntn_ctrl machine refine the ones of the top level specification. The boot event fixes the value of active_t and the run event builds the continuous function $f c$ with steps of duration $d t$. $f c$ becomes the function $\mathrm{nfc}$, acting until now+dt instant.

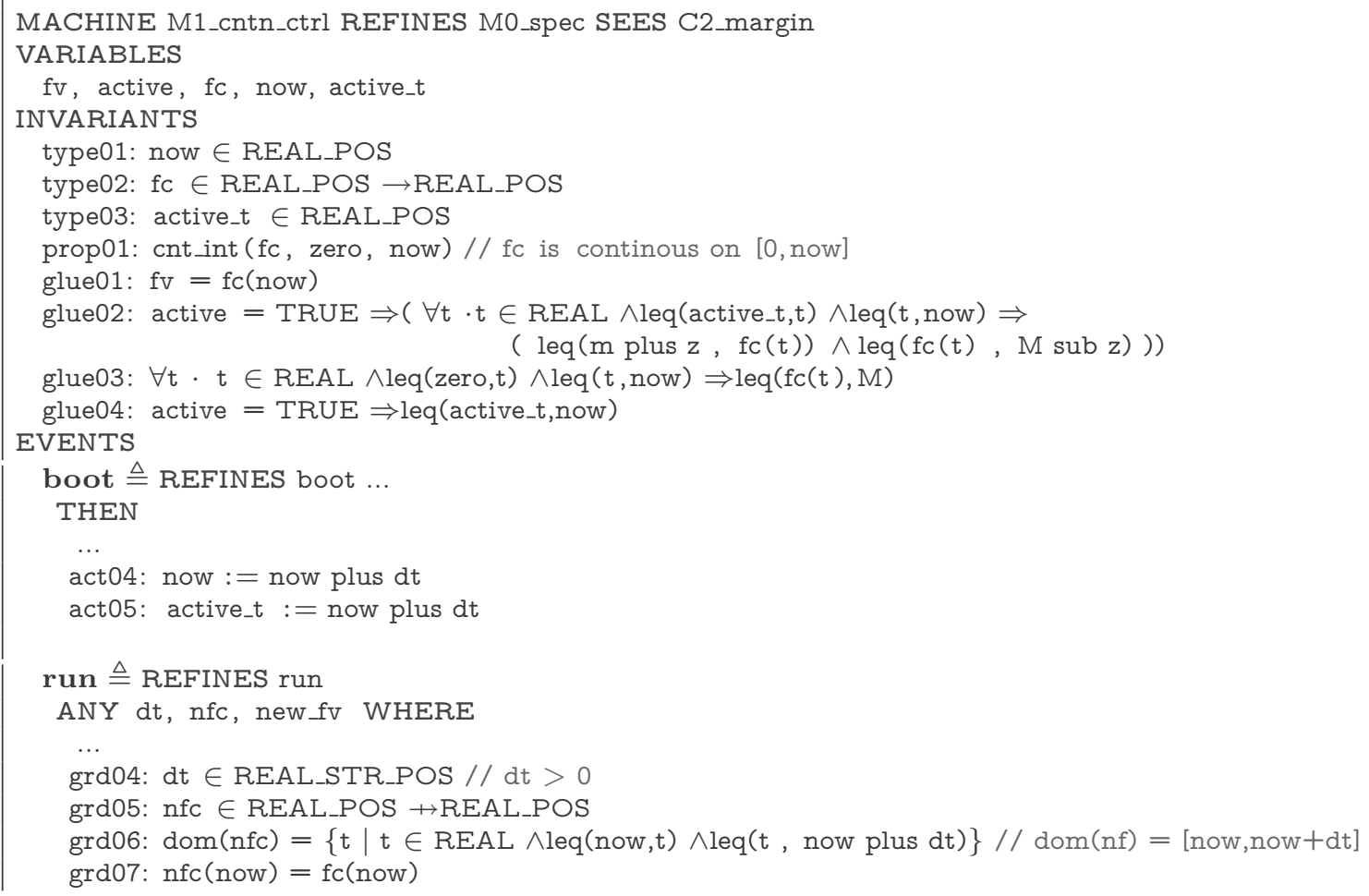




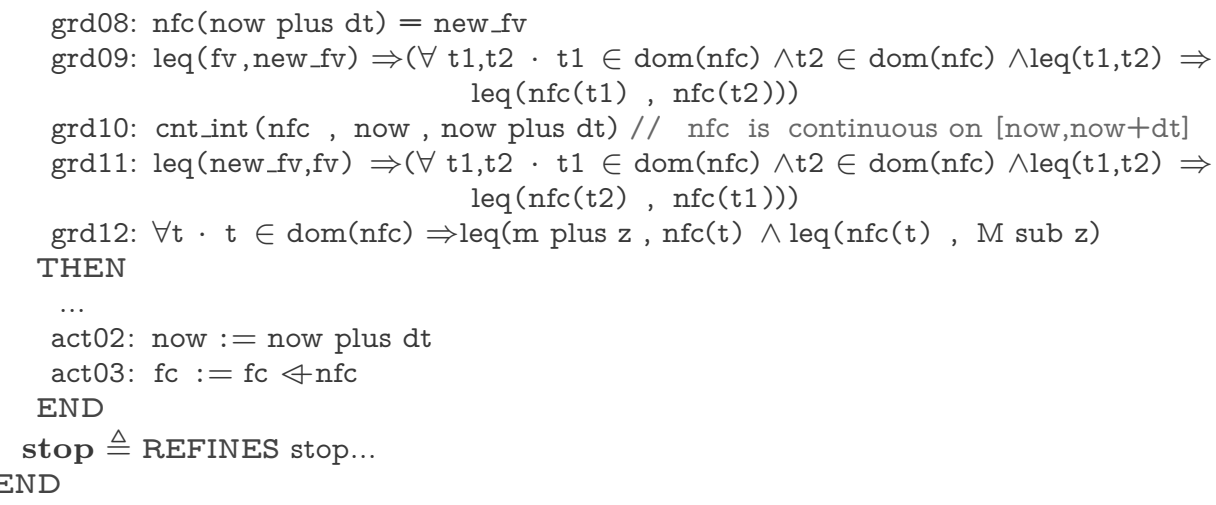

Listing 1.5. Extract of machine M1_cntn_ctrl

The current instant now is incremented by the step duration dt as well. The guards of the event run introduce the relevant conditions to trigger this event.

Note that during the time interval of the step, the function $\mathrm{fc}$ shall be continuous and monotonic so as its value is never outside the safety corridor (grd09 to grd11). This condition is fundamental when the function is discretized. Thus, grd09 through grd12 guarantee the requirement Req2.2 and are of particular importance when discretizing.

\subsection{The Second Refinement: Introducing Discrete Representation}

This refinement introduces the discretization function fd corresponding to the continuous function $f c$ on each discrete observed instants. This fundamental property corresponds to requirement Req2.2 of table 5 . It is expressed by the gluing invariants between the continuous controller and the discrete controller. It links the continuous $f_{c}$ and and discrete $f_{d}$ functions by the property $\forall n \in 0 \ldots i, f_{c}(n \times \delta t)=f_{d}(n)$ and is represented by invariant glue01.

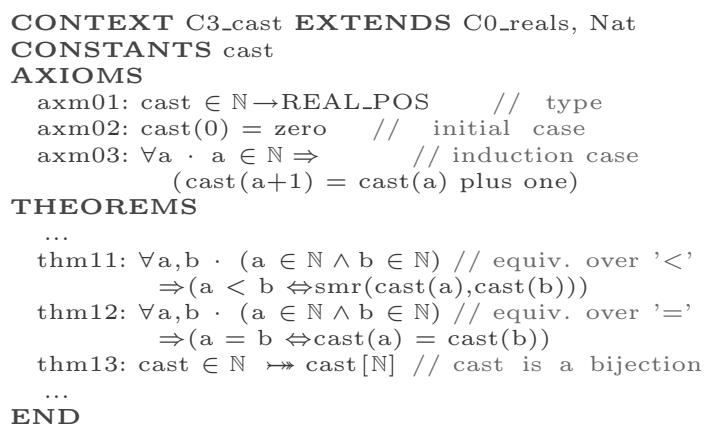

Listing 1.6. Definition and properties of the cast function

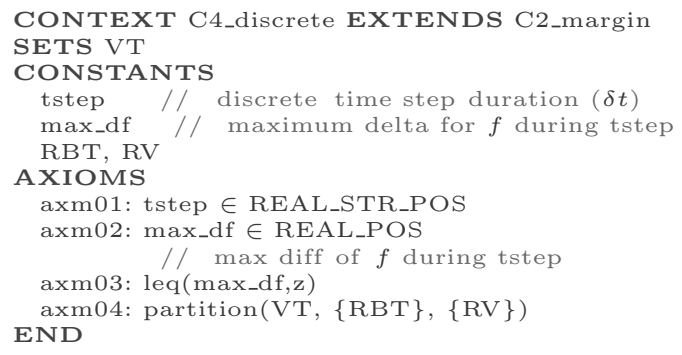

Listing 1.7. Extract of context C4_discrete 


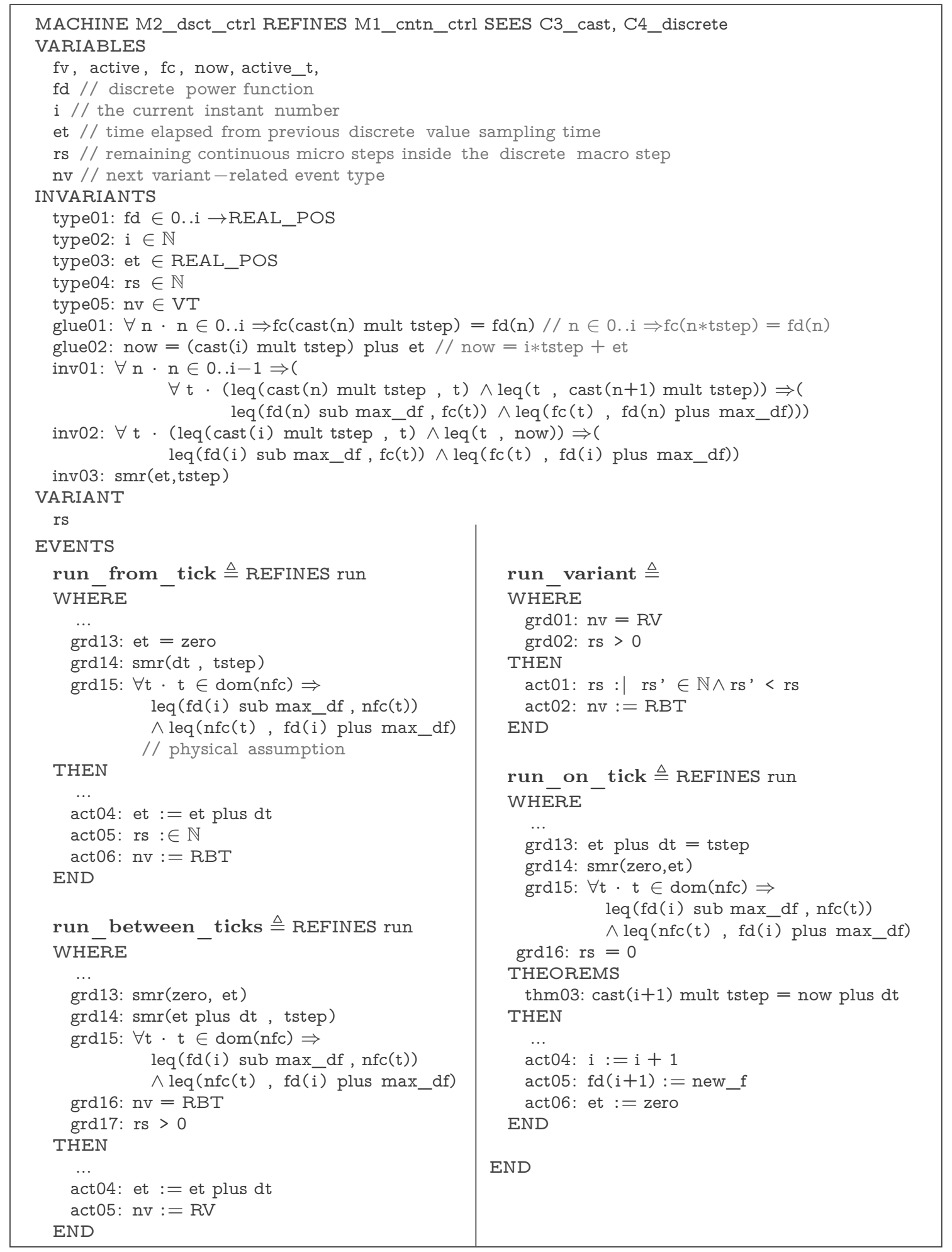

Listing 1.8. Extract of machine M2_dsct_ctrl 
Needed Theories. Two contexts are introduced. As mentioned in section 3 the first context C3_cast is a technical context related to casting reals and integers. For example, the invariant $\forall n \in 0 . . i, f_{c}(n \times \delta t)=f_{d}(n)$ corresponding to glue01 is written as $\forall n \cdot n \in 0$..i $\Rightarrow f c(\operatorname{cast}(n)$ mult tstep) $=f d(n)$.

Note that the context C3_cast extends the Nat context ${ }^{3}$ by Thai Son Hoang needed for handling inductive proofs on sets ${ }^{4}$. The last context C4_discrete introduces the discrete time macro steps duration tstep corresponding to $\delta t$ on figure 3 and the values RBT and RV to identify the different events corresponding to the run event. It also defines the max_df constant corresponding to the maximum evolution of the function in a macro step is never more that the margin z. This assumption usually comes from the conditions on the physical plant.

The Event-B Refinement with Discretization. The defined machine M2_dsct_ctrl produces the discrete behavior of the continuous function $\mathrm{fc}$ with the discrete function $\mathrm{fd}$ glued by the invariant glue01. The other invariants inv01 and inv02 preserve Req2.2 and inv03 states that the elapsed time et is less that the discrete time tstep. According to figure 3, three events for ROT, RBT and RFT are defined refine the run event. The run_from_tick (RFT) event starts the computation between two consecutive discrete values of function fd and fixes an arbitrary value of the variant rs.

The most interesting part in this machine relates to the run_between_tick (RBT) event which shall avoid the Zeno problem. For this purpose, each time this event is active, it triggers the event run_variant which decreases the variant. Once, this variant reaches the value 0 , the run_on_tick (ROT) event is triggered to compute the final value corresponding to next discrete value of the function $\mathrm{fd}$. Note that the guard grd15 is fundamental to guarantee that the values do not exit the safety corridor. This assumption relates to the physical plant.

\subsection{Proofs Statistics}

All these models have been encoded within the Rodin Platform [2]. As shown on table 6 , the main machine and the refinement led to 265 proof obligations.

Table 6. Rodin proofs statistics

\begin{tabular}{|l|c|c|c|}
\hline Event-B model & Automatic proofs & Interactive proofs & Total \\
\hline C0_reals & 1 & 29 & 30 \\
\hline C1_corridor & 0 & 6 & 6 \\
\hline C2_margin & 0 & 10 & 10 \\
\hline C3_cast & 11 & 26 & 37 \\
\hline C4_discrete & 0 & 1 & 1 \\
\hline M0_spec (top-level) & 11 & 6 & 17 \\
\hline M1_cntn_ctrl (1st ref.) & 22 & 51 & 73 \\
\hline M2_dsct_ctrl (2nd ref.) & 22 & 67 & 89 \\
\hline Total & 67 & 198 & 265 \\
\hline
\end{tabular}

\footnotetext{
${ }^{3}$ http://sourceforge.net/p/rodin-b-sharp/mailman/message/30378566/

${ }^{4}$ induction: $\forall \mathrm{S} \cdot \mathrm{S} \subseteq \mathbb{N} \wedge 0 \in \mathrm{S} \wedge(\forall \mathrm{x} \cdot \mathrm{x} \in \mathrm{S} \Rightarrow \mathrm{x}+1 \in \mathrm{S}) \Rightarrow \mathbb{N} \subseteq \mathrm{S}$
} 
67 were proved automatically and 198 needed numerous interactive proof steps. The interactive proofs mainly relate to the use of the Theory plugin for handling the reals. The lack of dedicated heuristics due to the representation of reals as an abstract data type, and not as a native type led to more interactive proofs.

\section{Related Works and Applications}

Two kinds of approaches for modeling hybrid systems can be distinguished. The first one relies on hybrid automata. They are mainly analyzed and verified by model checking [5]. Tools like HyTech [18] have succeeded in analyzing complex hybrid systems. While this approach enables automatic verification, it requires elaborate optimization techniques in order to handle the state space explosion as well as symbolic parameters and non-linear equations. To address these problems, logical analysis of hybrid automata brought interesting results [19]. They address classes of automata. The second kind of approaches relates to analysis of hybrid programs. One of the most successful tool is KeYmaera by Platzer et al. [22]. This tool is dedicated to hybrid system modeling and verification. It is equipped with an interactive theorem prover. Compared to Event-B, it does not provide a built-in refinement development operator. In the meantime, other approaches use Event-B to model hybrid systems. The work initiated in [23], and pursued in [12] proposes to model first the discrete events of a hybrid systems and then refine each event by introducing the continuous elements. It includes the use of a "now" variable, a "click" event that jumps in time to the next instant where an event can be triggered and simulated real numbers. In our proposal, we use this notion of "now" variable on dense time. Time jumps are encoded by the events. We use mathematical reals thanks to the latest developments of the Rodin Platform. Moreover, compared to [23], we have another refinement that introduces discretization of continuous elements. However, [23] incorporate analytical results from the study of differential equations into the Event-B models through the complementary use of Matlab/Simulink. The second proposed approach based on Event-B, initiated by Banach, is Hybrid Event-B [8]. This is an extension of Event-B which includes pliant events [7] as a way to model continuous behavior, allowing the direct use of differential equations in the modeling. However, there is no tool currently supporting this extension whereas our approach enabled us to develop and prove the models using available tools. Banach also worked on similar topics with ASM $[9,10]$. In our development we use reals defined by a minimal set of axioms. We do not use floating-point numbers, they may be introduced in a further refinement which is out of the scope of this paper. So, we are not exploiting the results from automated verification tools on floating-point numbers [21]. Static analysis [16] or abstract interpretation [14] (with tools such as Astrée [15]) have proved very powerful to analyze such programs. Our approach is at a modeling level. Moreover, the set of axioms for reals in the Theory plug-in we have used does not define reals in a constructive manner. So, we were not able to use the results obtained by the Coq [11] advanced proof tactics on reals. Indeed, our proofs have been discharged using the interactive prover of Rodin, leading to a large proof effort. 


\section{Conclusion}

The development of cyber-physical systems needs to handle the behavior of the physical plant (environment). This behavior is usually described by continuous functions producing feedback information to the controller, which in turns produces orders to the actuators. In this paper, we have shown that it is possible to compose the development of both a controller and the corresponding behavior of the physical plant. The controller corresponds to a hybrid automaton. A simple one has been considered in this paper. It consists in booting, running and then stopping a physical plant (see figure 1). The main contribution of this paper concerns the synthesis of a discrete controller. We have shown that the synthesis of a correct-by-construction discretization of a continuous function associated to the behavior of a physical plant can be obtained by refinement. The proof of the preservation of the invariants gluing the continuous and discrete levels guarantees this correctness. We have introduced at the discrete level a variant guaranteeing that the model is Zeno-free. The Theory plug-in for the Rodin Platform and a theory of real numbers have been used to model continuous functions. To the best of our knowledge, this is the first attempt to model continuous controller discretization with the Event-B method and mathematical reals.

As future work, we plan to address more complex hybrid automata by generalizing the approach presented in this paper. A particular case we expect to study relates to the system substitution in case of failure for example, already addressed in the discrete case in [6]. Another research path concerns the refinement by floating point numbers as another discretization step. This refinement will use the intermediate value theorem as gluing invariant between the obtained discretization level and the floating point level. Finally, an effort should be devoted to handle more efficiently the complex proof process set up in this paper.

\section{References}

1. Abrial, J.-R.: Modeling in Event-B: System and Software Engineering, 1st edn. Cambridge University Press, New York, NY, USA (2010)

2. Abrial, J.-R., Butler, M., Hallerstede, S., Hoang, T.S., Mehta, F., Voisin, L.: Rodin: an open toolset for modelling and reasoning in Event-B. Int. J. Softw. Tools Technol. Transfer 12(6), 447-466 (2010)

3. Abrial, J.-R., Butler, M., Hallerstede, S., Leuschel, M., Schmalz, M., Voisin, L.: Proposals for mathematical extensions for Event-B. Technical report (2009)

4. Abrial, J.-R., Hallerstede, S.: Refinement, decomposition, and instantiation of discrete models: Application to Event-B. Fundamenta Informat. 77(1), 1-28 (2007)

5. Alur, R.: Formal verification of hybrid systems. In: Chakraborty, S., Jerraya, A., Baruah, S. K., Fischmeister, S. (eds.) Proceedings of the 11th International Conference on Embedded Software, EMSOFT - ESWeek, Taipei, Taiwan, October 9-14, 2011, pp. 273-278. ACM (2011)

6. Babin, G., At-Ameur, Y., Pantel, M.: Formal verification of runtime compensation of web service compositions: A refinement and proof based proposal with Event-B. In: International Conference on SCC 2015 IEEE, pp. 98-105, June 
7. Banach, R.: Pliant modalities in Hybrid Event-B. In: Liu, Z., Woodcock, J., Zhu, H. (eds.) Theories of Programming and Formal Methods. LNCS, vol. 8051, pp. 37-53. Springer, Heidelberg (2013)

8. Banach, R., Butler, M., Qin, S., Verma, N., Zhu, H.: Core Hybrid Event-B I: Single Hybrid Event-B machines. Science of Computer Programming (2015)

9. Banach, R., Zhu, H., Su, W., Huang, R.: Formalising the continuous/discrete modeling step. In: Derrick, J., Boiten, E.A., Reeves, S. (eds.) Proceedings 15th International Refinement Workshop, Refine 2011, Limerick, Ireland, 20th June 2011, volume 55 of EPTCS, pp. 121-138 (2011)

10. Banach, R., Zhu, H., Su, W., Wu, X.: ASM and controller synthesis. In: Derrick, J., Fitzgerald, J., Gnesi, S., Khurshid, S., Leuschel, M., Reeves, S., Riccobene, E. (eds.) ABZ 2012. LNCS, vol. 7316, pp. 51-64. Springer, Heidelberg (2012)

11. Boldo, S., Lelay, C., Melquiond, G.: Coquelicot: A user-friendly library of real analysis for Coq. Math. Comput. Sci. 9(1), 41-62 (2015)

12. Butler, M., Abrial, J.-R., Banach, R.: From Action Systems to Distributed Systems: The Refinement Approach, chapter Modelling and Refining Hybrid Systems in Event-B and Rodin, p. 300. Taylor \& Francis, February 2016

13. Butler, M., Maamria, I.: Practical theory extension in Event-B. In: Liu, Z., Woodcock, J., Zhu, H. (eds.) Theories of Programming and Formal Methods. LNCS, vol. 8051, pp. 67-81. Springer, Heidelberg (2013)

14. Cousot, P., Cousot, R.: Abstract interpretation: A unified lattice model for static analysis of programs by construction or approximation of fixpoints. In: Proceedings of the 4th ACM POPL 1977, pp. 238-252, New York, NY, USA. ACM (1977)

15. Cousot, P., Cousot, R., Feret, J., Mauborgne, L., Miné, A., Monniaux, D., Rival, X.: The ASTRÉE analyzer. In: Sagiv, M. (ed.) ESOP 2005. LNCS, vol. 3444, pp. 21-30. Springer, Heidelberg (2005)

16. Goubault, É.: Static analyses of the precision of floating-point operations. In: Cousot, P. (ed.) SAS 2001. LNCS, vol. 2126, p. 234. Springer, Heidelberg (2001)

17. Henzinger. T. A.: The theory of hybrid automata. In: Inan, M.K., Kurshan, R.P. (eds.) Verification of Digital and Hybrid Systems, volume 170 of NATO ASI Series, pp. 265-292. Springer-Verlag (2000)

18. Henzinger, T.A., Ho, P.-H., Wong-Toi, H.: Hytech: A model checker for hybrid systems. International Journal on STTT 1(1-2), 110-122 (1997)

19. Ishii, D., Melquiond, G., Nakajima, S.: Inductive verification of hybrid automata with strongest postcondition calculus. In: Johnsen, E.B., Petre, L. (eds.) IFM 2013. LNCS, vol. 7940, pp. 139-153. Springer, Heidelberg (2013)

20. Lee, E.A., Seshia, S.A.: Introduction to Embedded Systems - A Cyber-Physical Systems Approach. LeeSeshia.org, edition 1.5 edition (2014)

21. Muller, J.-M., Brisebarre, N., de Dinechin, F., Jeannerod, C.-P., Lefévre, V., Melquiond, G., Revol, N., Stehlé, D., Torres, S.: Handbook of Floating-Point Arithmetic. Birkhäuser (2010)

22. Platzer, A.: Logical Analysis of Hybrid Systems: Proving Theorems for Complex Dynamics. Springer-Verlag, Heidelberg (2010)

23. Su, W., Abrial, J.-R., Zhu, H.: Formalizing hybrid systems with Event-B and the Rodin platform. Science of Computer Programming, 94, Part 2:164-202 (2014) 